\title{
Finite Time Controller for Point Stabilization of a Spherical Underwater Roving Robot
}

\author{
Zhimin Liu, Hanxu Sun, Yansheng Li, Qingxuan Jia, and Ming Chu \\ Institute of Automation, Beijing University of Posts and Telecommunications, Beijing 100876, China \\ Correspondence should be addressed to Zhimin Liu; qiierrsann@163.com
}

Received 21 April 2016; Revised 25 July 2016; Accepted 8 August 2016

Academic Editor: Ricardo Aguilar-López

Copyright ( 2016 Zhimin Liu et al. This is an open access article distributed under the Creative Commons Attribution License, which permits unrestricted use, distribution, and reproduction in any medium, provided the original work is properly cited.

\begin{abstract}
The finite time controller is proposed to solve the point stabilization problem for a novel underwater spherical roving robot (BYSQ3 ) in two-dimensional space. The finite time design scheme is a new method; the main advantage of this control scheme is that it can steer the robot to the origin in fast converging times without excessive control effort. Firstly, the physical prototype of BYSQ-3 is introduced and the equations describing the kinematics and dynamics of BYSQ-3 are established. Secondly, the finite time controller is constructed based on the backstepping method; the explicit form of the finite time controller is more concise compared with the other finite time controllers; there is no virtual input in the design process and the stability analysis is simple; the designed controller is easy for engineering implementation. Thirdly, the hydrodynamic characteristics is analyzed by CFD simulation; the simulation and experiment results are presented to validate the shorter convergence time and better stability character of the controller.
\end{abstract}

\section{Introduction}

Over the last two decades, the underwater autonomous vehicles (UAVs) have become a necessity for investigating and exploring the ocean resources. At the same time, the UAVs can undertake surveillance missions or mine laying and disposal operations with low cost [1].

The UAV under consideration in this paper is BYSQ-3, a roving UAV developed at Beijing University of Posts and Telecommunications (BUPT) (see Figure 1). A spherical hull is adopted due to its outstanding water pressure resistance and flexibility. The primary roles of the BYSQ-3 are deepsea detection or operation tasks. To carry out the tasks, it is necessary to stabilize the robot at a final target point with a desired attitude, that is, point stabilization problems. The point stabilization control plays a very significant role for UAVs to implement abroad range of applications [2]. Generally, the UAVs are underactuated systems equipped with fewer actuators than degrees of freedom; it is difficult to command accelerations in all DOF simultaneously [3]. Furthermore, due to the strongly coupled and intrinsic nonlinear nature of the UAVs' dynamical equations, the control of UAVs has become a great challenge attracting more attention from both marine technology and control engineering communities [4].

Because of the limitations of Brockett's theory [5], there is no pure state feedbacks that can stabilize the underactuated UAV s at a fixed-point asymptotically. Thus, the discontinuous feedback laws or time-varying continuous feedback laws are usually used to solve the point stabilization problems. In [6], the switching time-invariant control scheme was proposed for underactuated surface vehicles which can steer the vehicles to the desired target point which is asymptotically stable. In [7], discontinuous two-stage control laws based on the terminal sliding mode were proposed to solve the point stabilization problems. In [8], it was shown that the underactuated autonomous vehicles dynamical systems were small-time locally controllable and strongly accessible at origin and then the time-invariant discontinuous control laws were constructed to stabilize the system to the origin asymptotically. In [9], by using the coordinate transformations, the whole dynamical system could be transformed into a cascade system and then it was reduced to a thirdorder chained form; the controller was constructed based on the backstepping approach. In [10], a set-point controller was proposed for UAVs in transformed equations of motion, 
the system was decoupled and each quasi-velocity could be regulated separately. In $[11,12]$, a logic-based feedback hybrid controller was proposed which exhibited a simple structure and ensured that the system converges to an arbitrarily small neighborhood of the origin asymptotically. There are several other control schemes proposed for the point stabilization problems; see [13-16].

However, the control schemes proposed in the aforementioned literature have at best exponential convergence rate to an equilibrium; in other words, to steer the vehicles to a final target point with a desired orientation in finite time is difficult. In fact, there are many time sensitive tasks, for example, rendezvous missions for mobile agents, underwater search and rescue, and surveillance and detection. It is desirable that the underwater tasks can be accomplished quickly in finite time rather than merely over the infinite horizon. The finite time control laws have the good performance such as higher accuracies, faster convergence rate, and better disturbance rejection properties. Therefore, the design of finite time control laws for nonlinear underactuated systems have attracted considerable attention from the control field. For example, in [17], the point stabilization problem of the underwater robot was solved by using a sequential series of switched control laws, each of which can achieve a certain objective; finally the system can be steered to the origin in finite time. In [18], the trajectory tracking control problem for underactuated UAVs was addressed, the finite time tracking control laws were developed, and the tracking errors converged to the origin in finite time but the yaw angular $r$ was BIBO stabilization. In [19], the underactuated nonholonomic systems with chained form were investigated by output feedback control law; the designed controller rendered the state variables to zero within finite time. In [20], a class of high-order nonlinear systems' point stabilization problem was investigated; by combining the sign function with adaptive technique, the convergent time can be adjusted by preassigning the design parameter. The other results associated with this field; see [21-24].

The adding-a-power-integrator technique has been used in many literatures. By using the adding-a-power-integrator technique, combining with other techniques, for example, the related adaptive technique in [25], a scaling gain and appropriate Lyapunov-Krasovskii functional in [26, 27], the homogeneous domination approach in [28], and the sign functions in [29], a class of uncertain high-order nonlinear systems with time-varying delay system can be rendered globally asymptotically stable; however, the aforementioned results are merely asymptotic. In [30], a class of nonholonomic systems in chained form which can model mobile robots and wheeled vehicles was studied, the finite time state feedback controller was addressed; however, the method requires the sway velocity satisfying the first-order nonholonomic constraints (the sway velocity must be zero); it cannot be applied to the control of UAVs.

Motivated by [25-31], the finite time controller is constructed based on the adding a power integrator technique and backstepping method; the explicit form of the finite time controller is more concise compared with the finite time controller proposed in $[30,32,33]$; the coordinate transformations are introduced and the system is decoupled and then the designed controller can steer the state variables to zero fast. Compared with the other traditional approaches, the proposed approaches have shorter convergence time and it is the energy saving control strategy.

The paper is organized as follows. Section 2 introduces the preliminaries and problem formulation. Section 3 presents the process of the controller design. Section 4 contains the simulation results of the proposed control strategy and the comparison results by the designed controller and the controller in [17]. Section 5 depicts and analyzes the experiment results. Section 6 provides the conclusions.

\section{Preliminaries and Problem Formulation}

\subsection{Preliminaries}

Definition 1 (see [34]). Consider the system given by

$$
\dot{x}(t)=f(x(t))=\left[f_{1}(x), f_{2}(x), \ldots, f_{n}(x)\right]^{T},
$$

where $x(t) \in D C R^{n},\left(t \in\left[t_{0}, T\right), T \leq \infty\right)$ is the state vector, $f(0)=0, D$ is an open set, $0 \in D$, and $f(x(t))$ is continuous on $D$. Let the origin $x(t)=0$ be an equilibrium point of system (1); then the origin is said to be finite time stable if an open neighborhood $U \in D$ of the origin exists.

(i) Finite Time Convergence. That is, for every $x \in U \backslash\{0\}$, a settling-time function $T: U \backslash\{0\} \rightarrow R^{+}$exists such that, for all $x \in U \backslash\{0\}, s(x, t) \in U \backslash\{0\}$ is defined on $[0, T(x))$, nonzero, and satisfies $\lim _{t \rightarrow T(x)} s(t, x)=$ 0 for all $t \in[0, T(x))$; if $U=R^{n}$, the zero solution $x(t)=0$ of system (1) is said to be globally finite time stable.

(ii) Stability in the Sense of Lyapunov. That is, for all $t \epsilon$ $[0, T(x))$ and for any $\varepsilon>0, \delta(t, \varepsilon)>0$ exists such that, for every $\|x\|<\delta,\|s(t, x)\|<\varepsilon$.

Definition 2 (see [34]). Consider the nonlinear dynamical system (1); assume that the origin $x(t)=0$ be an equilibrium point, if there exists a continuously differentiable function $V(x)$, and the following statements are satisfied:

(i) $V(x)$ is positive definite.

(ii) Two real numbers $c>0,0<\alpha<1$ and an open neighborhood $U_{0} \in D$ of the origin exist such that

$$
\dot{V}(x)+c(V(x))^{\alpha} \leq 0 ;
$$

then the origin $x(t)=0$ is said to be a finite time stable equilibrium of (1) and the settling-time

$$
T(x) \leq \frac{1}{c(1-\alpha)}(V(x))^{1-\alpha} .
$$

Lemma 3 (see [32]). For any real numbers $x$ and $y$, let $c, d$ be two positive real numbers, $0<q=q_{1} / q_{2}<1$, and $q_{1}, q_{2}$ are two coprime positive odds; the following inequality holds:

$$
\begin{aligned}
|x|^{c}|y|^{d} & \leq \frac{c|x|^{c+d}}{(c+d)}+\frac{d|y|^{c+d}}{(c+d)}, \\
\left|\left(x^{q}-y^{q}\right)\right| & \leq 2^{1-q}|x-y|^{q} .
\end{aligned}
$$



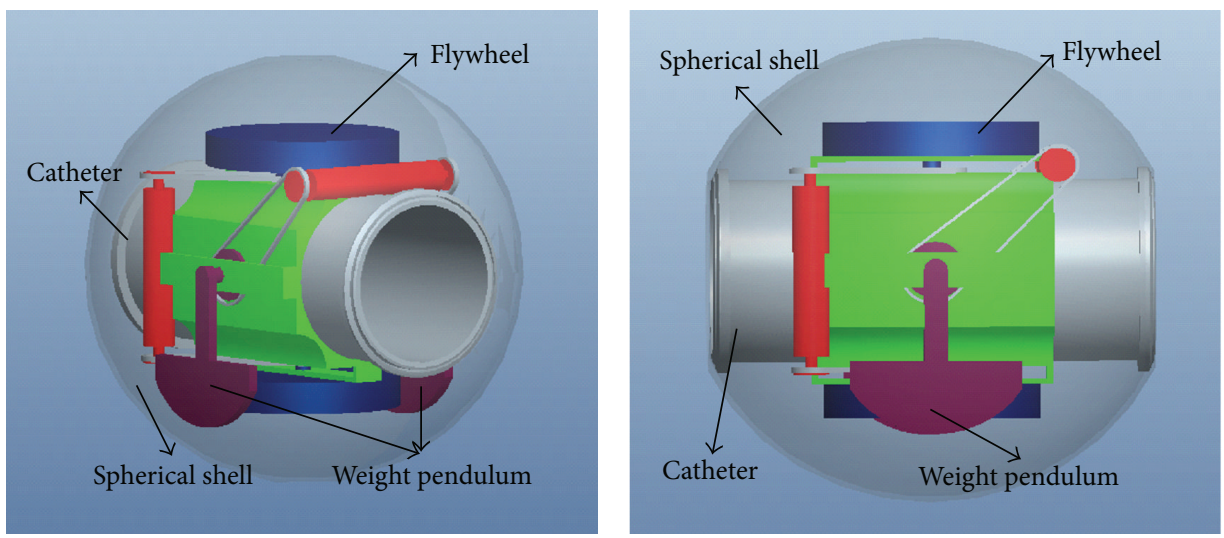

FIGURE 1: Three-dimensional structure chart.

Throughout this paper, the above definitions and lemma are used to help us to design and analyze the finite time stability controller for the point stabilization problems of BYSQ-3.

\subsection{Inner Structure and Dynamics of BYSQ-3. BYSQ-3 is the} third generation underwater robot of BUPT; it adopts spherical hull; the general structure of BYSQ-3 mainly consists of a single propeller, the heavy pendulums, and a flywheel (see Figures 1 and 8). The catheter is fixed to the spherical shell and the propeller is mounted in the middle of the catheter; the sleeve (or long axis) is mounted on the outer wall of the catheter and it can rotate around the catheter; the short axis mechanism and the flywheel rotation axis are fixed to sleeve and perpendicular to the sleeve. The robot adjusted its attitude by using the heavy pendulums and the flywheel. The heavy pendulums can rotate around the long or short axis; thus, the robot's roll or pitch angle can change. Driving the flywheel motor, the robot's yaw angle can change. The single propeller provides the thrust to make the robot move forward. The driving motors and control module are sealed inside the spherical shell. The three-dimensional structure of BYSQ-3 is shown in Figure 1. The BYSQ-3 is equipped with propeller and flywheel for surge and yaw motion and there is no direct actuators for sway motion, so it is said to be underactuated.

In this paper, we consider BYSQ-3 as a rigid body; the inertial reference and the body-fixed reference are depicted in Figure 2. The equations describing the kinematics and dynamics of BYSQ-3 can be written as follows (see [6]):

$$
\begin{aligned}
& \dot{x}=u \cos \varphi-v \sin \varphi, \\
& \dot{y}=u \sin \varphi+v \cos \varphi, \\
& \dot{\varphi}=r, \\
& \dot{u}=\frac{\left(m_{22} v r-d_{11} u+\tau_{1}\right)}{m_{22}}, \\
& \dot{v}=-\frac{\left(m_{11} u r+d_{22} v\right)}{m_{22}}, \\
& \dot{r}=\frac{\left(\left(m_{11}-m_{22}\right) u v-d_{33} r+\tau_{3}\right)}{m_{33}},
\end{aligned}
$$

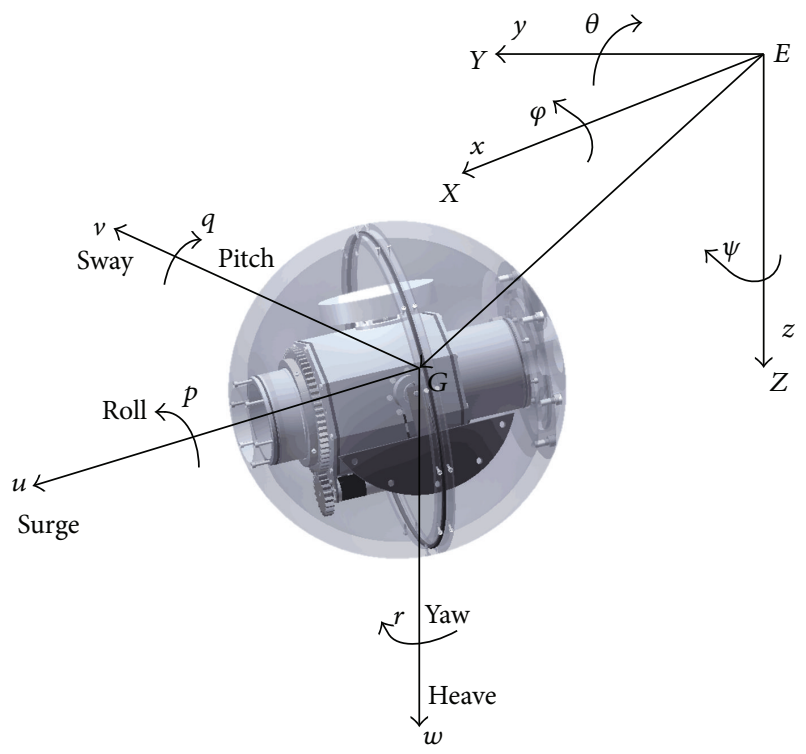

Figure 2: The inertial and the body-fixed reference frames.

where $x, y$ represent the positions and $\varphi$ is the yaw angle representing the orientation of BYSQ-3 in the inertial frame; $u, v$ represent linear velocities and $r$ represents angular velocity of BYSQ-3 in the body-fixed frame; $m_{i i}, i=1,2,3$, represent the inertia including added masses, $d_{i i}, i=1,2,3$, represent the drag coefficients of BYSQ-3; $\tau_{1}, \tau_{3}$ are the forces of the thruster and torque generated by flywheel motor. No motors are aligned in the sway direction. The 3 DOFs' horizontal motion must be controlled by the forward thrust control $\tau_{1}$ and the rotating torque $\tau_{3}$. So, (5) is an underactuated control system.

Assumption 4. (i) The center of gravity coincides with the center of buoyancy. (ii) The spherical shell is perfectly spherical and the mass distribution of BYSQ- 3 is homogeneous. (iii) The heave, pitch, and roll movements are neglected and the effects of wave, wind, and current are ignored.

2.3. Control Objectives. The control objective is to move the BYSQ-3 from one initial motion state to another desired 
motion state. Without loss of generality, we describe the problem as the stabilization of a nonlinear underactuated system to the origin from a nonzero initial configuration.

\section{Design of the Control System}

To facilitate the design of the control laws, we will convert system (5) to the following suitable form by using the coordinate and input transformations, which have been used in [6]:

$$
\begin{aligned}
& \left(\begin{array}{l}
z_{1} \\
z_{2} \\
z_{3} \\
z_{4} \\
z_{5} \\
z_{6}
\end{array}\right) \\
& =\left(\begin{array}{cccccc}
\cos \varphi & \sin \varphi & 0 & 0 & 0 & 0 \\
-\sin \varphi & \cos \varphi & 0 & 0 & \frac{m_{22}}{d_{22}} & 0 \\
0 & 0 & 1 & 0 & 0 & 0 \\
-\cos \varphi & -\sin \varphi & 0 & \frac{-m_{11}}{d_{22}} & 0 & 0 \\
0 & 0 & 0 & 0 & 1 & 0 \\
0 & 0 & 0 & 0 & 0 & 1
\end{array}\right)\left(\begin{array}{l}
x \\
y \\
\varphi \\
u \\
v \\
r
\end{array}\right), \\
& F_{1}=\frac{\left(\left(d_{11}-d_{22}\right) u-d_{22} z_{2} z_{6}-\tau_{1}\right)}{d_{22}}, \\
& F_{2}=\frac{\left(\left(m_{11}-m_{22}\right) u v-d_{33} r+\tau_{3}\right)}{m_{33}} \text {; }
\end{aligned}
$$

it is easy to see that the coordinate transformations are invertible, and $F_{1}, F_{2}$ can be seen as the new control inputs; then, system (5) is converted to the following form:

$$
\begin{aligned}
& \dot{z}_{1}=-a z_{1}+z_{2} z_{6}-a z_{4}-b z_{5} z_{6}, \\
& \dot{z}_{5}=c\left(z_{1}+z_{4}\right) z_{6}-c z_{5}, \\
& \dot{z}_{2}=z_{4} z_{6}, \\
& \dot{z}_{3}=z_{6}, \\
& \dot{z}_{4}=F_{1} \\
& \dot{z}_{6}=F_{2},
\end{aligned}
$$

where

$$
\begin{aligned}
& a=\frac{d_{22}}{m_{11}}, \\
& b=\frac{m_{22}}{d_{22}}, \\
& c=\frac{d_{22}}{m_{22}} .
\end{aligned}
$$

It had been proved in [6] that system (7) is globally uniformly asymptotically stabilized, if system (8) can be globally uniformly asymptotically stabilized by any control law.

Given that the initial value of $z_{6}$ satisfies $z_{6}(0) \neq 0$, the design of the control laws can be divided into three stages.

Stage 1. By using the following control laws, we can regulate the state variables $z_{2}, z_{4}$ to the origin in finite time $T_{1}$. To realize the objective, we set $F_{2}=0$.

Theorem 5. The nonlinear subsystem of (8),

$$
\begin{aligned}
& \dot{z}_{2}=z_{4} z_{6}, \\
& \dot{z}_{4}=F_{1},
\end{aligned}
$$

can be stabilized by the following finite time control laws:

$$
\begin{aligned}
& F_{1}=\frac{-a_{1}\left(\left(z_{4} z_{6}\right)^{p_{1}}+c_{1}^{p_{1}} z_{2}\right)^{\left(1-\delta_{1}\right) / p_{1}}}{z_{6}}, \\
& F_{2}=0
\end{aligned}
$$

$$
\begin{aligned}
& \text { where } a_{1} \geq\left(\left(2 p_{1}-1\right) / p_{1}\right) c_{1}^{1+p_{1}}\left(2^{1-1 / p_{1}}+2^{2-2 / p_{1}} / c_{1}+\varepsilon_{1}\right), \\
& c_{1}=2^{p_{1}-1 / p_{1}}+\varepsilon_{1}, \\
& \varepsilon_{1}>0,0<\delta_{1}<1, p_{1}=1+\delta_{1}, \delta_{1}=\frac{w_{1}}{w_{2}},
\end{aligned}
$$

where $w_{1}$ is a positive and even number and $w_{2}$ is a positive and odd number.

Proof. Let $x_{1}=z_{2}, x_{2}=z_{4} z_{6}, F_{1}=u / z_{6}$; then system (11) can be converted to

$$
\begin{aligned}
& \dot{x}_{1}=x_{2}, \\
& \dot{x}_{2}=z_{6} F_{1}=u .
\end{aligned}
$$

Remark 6. If the states $x_{1}, x_{2}$ can be stabilized in finite time, from $x_{1}=z_{2}, x_{2}=z_{4} z_{6}\left(z_{6} \neq 0\right)$, we know that the states $z_{2}, z_{4}$ can be stabilized in finite time. In this stage, $F_{2}=0$, $\dot{z}_{6}=F_{2}$; hence, $z_{6}(t)=z_{6}(0) \neq 0$.

By using Definitions 1 and 2, we describe that system $\left(11^{*}\right)$ can be stabilized in finite time by

$$
u=-a_{1}\left(\left(x_{2}\right)^{p_{1}}+b^{p_{1}} x_{1}\right)^{\left(1-\delta_{1}\right) / p_{1}},
$$

where

$$
\begin{aligned}
& a_{1} \geq\left(\frac{2 p_{1}-1}{p_{1}}\right) c_{1}^{1+p_{1}}\left(2^{1-1 / p_{1}}+\frac{2^{2-2 / p_{1}}}{c_{1}}+\varepsilon_{1}\right), \\
& c_{1}=2^{p_{1}-1 / p_{1}}+\varepsilon_{1}, \\
& \varepsilon_{1}>0,0<\delta_{1}<1, p_{1}=1+\delta_{1}, \delta_{1}=\frac{w_{1}}{w_{2}} .
\end{aligned}
$$


Define the candidate Lyapunov function:

$$
V_{1}\left(x_{1}\right)=\frac{x_{1}^{2}}{2} .
$$

Its time derivative along system $\left(11^{*}\right)$ renders to

$$
\dot{V}_{1}\left(x_{1}\right)=\left(x_{1} x_{2}-x_{1} x_{2}^{*}\right)+x_{1} x_{2}^{*} .
$$

Let

$$
x_{2}^{*}=-c_{1} x_{1}^{d_{1}-1}, \quad c_{1}>0, d_{1}=1+\frac{1}{p_{1}} ;
$$

then

$$
\begin{aligned}
\dot{V}_{1}\left(x_{1}\right) & =\left(x_{1} x_{2}-x_{1} x_{2}^{*}\right)+x_{1} x_{2}^{*} \\
& =\left(x_{1} x_{2}-x_{1} x_{2}^{*}\right)-c_{1} x_{1}^{d_{1}},
\end{aligned}
$$

and we have

$$
\dot{V}_{1}\left(x_{1}\right) \leq-c_{1} x_{1}^{d_{1}}+\left|x_{1} x_{2}-x_{1} x_{2}^{*}\right| .
$$

Define the candidate Lyapunov function

$$
\begin{aligned}
V_{2}\left(x_{1}, x_{2}\right) \\
=\frac{x_{1}^{2}}{2} \\
\quad+\frac{1}{\left(2-1 / p_{1}\right) c_{1}{ }^{1+p_{1}}} \int_{x_{2}^{*}}^{x_{2}}\left(s^{p_{1}}-x_{2}^{* p_{1}}\right)^{2-1 / p_{1}} d s .
\end{aligned}
$$

From [32], we know the following.

$V_{2}\left(x_{1}, x_{2}\right)$ is time derivative and positive definite and

$$
\begin{aligned}
\dot{V}_{2}\left(x_{1}, x_{2}\right)= & \dot{V}_{1}\left(x_{1}\right)+\frac{1}{\left(2-1 / p_{1}\right) c_{1}{ }^{1+p_{1}}} \theta^{2-1 / p_{1}} u \\
& +\frac{1}{c_{1}} x_{2} \int_{x_{2}^{*}}^{x_{2}}\left(s^{p_{1}}-x_{2}^{* p_{1}}\right)^{1-1 / p_{1}} d s,
\end{aligned}
$$

where

$$
\theta=x_{2}^{p_{1}}-x_{2}^{* p_{1}}=x_{2}^{p_{1}}+c_{1}^{p_{1}} x_{1} .
$$

From (19) and (21), we have

$$
\begin{aligned}
\dot{V}_{2}\left(x_{1}, x_{2}\right) \leq & -c_{1} x_{1}^{d_{1}}+\left|x_{1} x_{2}-x_{1} x_{2}^{*}\right| \\
& +\frac{1}{\left(2-1 / p_{1}\right) c_{1}^{1+p_{1}}} \theta^{2-1 / p_{1}} u \\
& +\frac{1}{c_{1}}\left|x_{2}\right| \theta^{1-1 / p_{1}}\left|x_{2}-x_{2}^{*}\right| .
\end{aligned}
$$

Using Lemma 3, we have

$$
\begin{aligned}
\left|x_{1} x_{2}-x_{1} x_{2}^{*}\right| & =\left|x_{1}\right|\left|x_{2}-x_{2}^{*}\right| \\
& =\left|x_{1}\right|\left|\left(x_{2}^{p_{1}}\right)^{1 / p_{1}}-\left(\left(x_{2}^{*}\right)^{p_{1}}\right)^{1 / p_{1}}\right| \\
& \leq 2^{1-1 / p_{1}}|\theta|^{1 / p_{1}}\left|x_{1}\right| \\
& \leq 2^{1-1 / p_{1}}\left(\frac{p_{1} x_{1}^{d_{1}}}{1+p_{1}}+\frac{\theta^{d_{1}}}{1+p_{1}}\right) .
\end{aligned}
$$

For

$$
\left|x_{2}\right|\left|x_{2}-x_{2}^{*}\right| \leq\left|x_{2}-x_{2}^{*}\right|\left|x_{2}-x_{2}^{*}\right|+\left|x_{2}^{*}\right|\left|x_{2}-x_{2}^{*}\right|,
$$

using Lemma 3 and (24) we have

$$
\begin{aligned}
\left|x_{2}-x_{2}^{*}\right|\left|x_{2}-x_{2}^{*}\right| & \leq 2^{2-2 / p_{1}}|\theta|^{2 / p_{1}}, \\
\left|x_{2}^{*}\right|\left|x_{2}-x_{2}^{*}\right| & \leq c_{1} 2^{1-1 / p_{1}}|\theta|^{1 / p_{1}}\left|x_{1}\right|^{1 / p_{1}} .
\end{aligned}
$$

Then, by using Lemma 3 and combining (23), (24), and (26), we obtain

$$
\begin{aligned}
\dot{V}_{2}\left(x_{1}, x_{2}\right) \leq & -c_{1} x_{1}^{d_{1}}+2^{1-1 / p_{1}}\left(\frac{p_{1} x_{1}^{d_{1}}}{1+p_{1}}+\frac{\theta^{d_{1}}}{1+p_{1}}\right) \\
& +\frac{1}{\left(2-1 / p_{1}\right) c_{1}^{1+p_{1}}} \theta^{2-1 / p_{1}} u \\
& +\frac{1}{c_{1}} 2^{2-2 / p_{1}} \theta^{d_{1}} \\
& +2^{1-1 / p_{1}}\left(\frac{x_{1}^{d_{1}}}{1+p_{1}}+\frac{p_{1} \theta^{d_{1}}}{1+p_{1}}\right) .
\end{aligned}
$$

Let

$$
c_{1}=2^{p_{1}-1 / p_{1}}+\varepsilon_{1}
$$

subsisting (11) into (27), we have

$$
\dot{V}_{2}\left(x_{1}, x_{2}\right) \leq-\varepsilon_{1} x_{1}^{d_{1}}-\varepsilon_{1} \theta^{d_{1}} .
$$

For

$$
\begin{aligned}
& V_{2}\left(x_{1}, x_{2}\right) \\
& \quad \leq \frac{x_{1}^{2}}{2}+\frac{1}{\left(\left(2 p_{1}-1\right) / p_{1}\right) c_{1}^{1+p_{1}}}\left|x_{2}-x_{2}^{*}\right| \theta^{2-1 / p_{1}},
\end{aligned}
$$

using Lemma 3, we have

$$
V_{2}\left(x_{1}, x_{2}\right) \leq \frac{x_{1}^{2}}{2}+\frac{2^{p_{1}-1 / p_{1}}}{\left(\left(2 p_{1}-1\right) / p_{1}\right) c_{1}^{1+p_{1}}} \theta^{2} .
$$

Let $\beta=\max \left(1 / 2,2^{p_{1}-1 / p_{1}} /\left(\left(2 p_{1}-1\right) / p_{1}\right) c_{1}^{1+p_{1}}\right)$; then $V_{2}\left(x_{1}\right.$, $\left.x_{2}\right) \leq \beta x_{1}^{2}+\beta \theta^{2}$.

Let $b=\varepsilon_{1} / 2 \beta^{\gamma}, \gamma=d_{1} / 2$, and $0<\gamma<1$; we have

$$
\dot{V}_{2}+b V_{2}^{\gamma} \leq-\frac{1}{2}\left(\varepsilon_{1} x_{1}^{d_{1}}-\varepsilon_{1} \theta^{d_{1}}\right) \leq 0 .
$$

Using Definition 2, system (10) is globally uniformly asymptotically stabilized.

Stage 2. In this stage, we regulate the state variables $z_{3}, z_{6}$ to the origin in finite time $T_{2}$; to realize the objective, we set $F_{1}=$ 0 .

The nonlinear subsystem of (8) is

$$
\begin{aligned}
& \dot{z}_{3}=z_{6}, \\
& \dot{z}_{6}=F_{2} ;
\end{aligned}
$$


it is a 2-order integrator system; to regulate $z_{3}, z_{6}$ to origin in finite time, we construct the following controller which can stabilize system (33) in finite time:

$$
\begin{aligned}
& F_{1}=0, \\
& F_{2}=-a_{2}\left(z_{6}^{p_{2}}+e^{p_{2}} z_{3}\right)^{\left(1-\delta_{2}\right) / p_{2}}, \\
& \quad t \geq T_{1} .
\end{aligned}
$$

Theorem 7. The switching controller

$$
\begin{array}{ll}
F_{1}=-a_{1}\left(x_{2}^{p_{1}}+b^{p_{1}} x_{1}\right)^{\left(1-\delta_{1}\right) / p_{1}}, & \\
F_{2}=0, & t<T_{1}, \\
F_{1}=0, & \\
F_{2}=-a_{2}\left(z_{6}^{p_{2}}+e^{p_{2}} z_{3}\right)^{\left(1-\delta_{2}\right) / p_{2}}, & \\
& t \geq T_{1}
\end{array}
$$

moves the states $z_{2}, z_{3}, z_{4}, z_{6}$ to zero in finite time $T_{1}+T_{2}$ and at the end of which we have $z_{2}(t)=z_{3}(t)=z_{4}(t)=z_{6}(t)=0$; the state variables $z_{1}, z_{5}$ are bounded and the closed-loop system is globally finite time stable.

Proof. From the above control laws, the state variables $z_{2}, z_{4}$ converge to zero at time $T_{1}$. When $t \geq T_{1}$, the change of $F_{2}$ affects the state variables $z_{3}, z_{6}$ while $z_{2}, z_{4}$ remain unaffected. Below we illustrate that there does not exist finite time escape phenomenon for $z_{3}, z_{6}$, when $t \in\left[0, T_{1}\right], F_{2}=0$; from $\dot{z}_{6}=F_{2}, z_{6}$ will be a constant value $\left(z_{6}(t)=z_{6}(0)\right)$ and from $\dot{z}_{3}=z_{6}$, we know $z_{3}$ exhibits a linear increase with time and is bounded. From the design of the control law, we have $z_{2}(t)=z_{3}(t)=z_{4}(t)=z_{6}(t)=0$, for all $t>T_{1}+T_{2}$. In fact, $z_{1}, z_{5}$ are bounded for all $t \in\left[0, T_{1}+T_{2}\right]$.

Define the Lyapunov function:

$$
V=\frac{d_{22}}{2 m_{22}} z_{1}^{2}+\frac{m_{22}}{d_{22}} z_{5}^{2}
$$

Then

$$
\begin{aligned}
\dot{V}= & \frac{d_{22}}{2 m_{22}} z_{1} \dot{z}_{1}+\frac{m_{22}}{d_{22}} z_{5} \dot{z}_{5} \\
= & \frac{d_{22}}{2 m_{22}} z_{1}\left(-\frac{d_{22}}{m_{11}} z_{1}-\frac{d_{22}}{m_{11}} z_{4}+z_{2} z_{6}-\frac{m_{22}}{d_{22}} z_{5} z_{6}\right) \\
& +\frac{m_{22}}{d_{22}} z_{5}\left(-\frac{d_{22}}{m_{22}} z_{5}-\frac{d_{22}}{m_{22}} z_{6}\left(z_{1}+z_{4}\right)\right) \\
= & -\frac{d_{22}^{2}}{m_{11} m_{22}} z_{1}^{2}-z_{5}^{2}-z_{1}\left(\frac{d_{22}^{2}}{m_{11} m_{22}} z_{4}-\frac{d_{22}}{m_{22}} z_{2} z_{6}\right) \\
& +z_{4} z_{5} z_{6} .
\end{aligned}
$$

From

$$
V=\frac{d_{22}}{2 m_{22}} z_{1}^{2}+\frac{m_{22}}{d_{22}} z_{5}^{2}
$$

we have

$$
\begin{aligned}
& \left|z_{1}\right| \leq \sqrt{\frac{2 m_{22}}{d_{22}}} \sqrt{V}, \\
& \left|z_{5}\right| \leq \sqrt{\frac{2 d_{22}}{m_{22}}} \sqrt{V} .
\end{aligned}
$$

Recalling that $z_{2}(t), z_{3}(t), z_{4}(t), z_{6}(t)$ are bounded for all $t \in$ $\left[0, T_{2}\right]$, we have

$$
\dot{V} \leq k_{1} V+k_{2}(t) \sqrt{V}
$$

where

$$
\begin{aligned}
k_{1}= & \max \left(-\frac{d_{22}^{2}}{m_{11} m_{22}},-1\right), \\
k_{2}(t)= & \left(\frac{d_{22}^{2}}{m_{11} m_{22}} z_{4}-\frac{d_{22}}{m_{22}} z_{2} z_{6}\right) \sqrt{\frac{2 m_{22}}{d_{22}}} \\
& +z_{4} z_{6} \sqrt{\frac{2 d_{22}}{m_{22}}} .
\end{aligned}
$$

Let $\delta=\sqrt{V}$; then

$$
\dot{\delta}=\frac{d \sqrt{V}}{d t} \leq \frac{k_{1}}{2} \delta+\frac{1}{2} k_{2}(t),
$$

so

$$
\delta(t) \leq e^{-(1 / 2) k_{1} T_{2}} \delta(0)+\frac{1}{2} \int_{0}^{T_{2}} e^{-(1 / 2) k_{1}\left(T_{2}-t\right)} k_{2}(t) d t
$$

Recalling that $k_{2}(t)$ is bounded, so $z_{1}, z_{5}$ are bounded for all $t \in\left[0, T_{2}\right]$.

Stage 3. From the above analysis, when $t>T_{1}+T_{2}$, the system becomes

$$
\begin{aligned}
& \dot{z}_{1}=-a z_{1}, \\
& \dot{z}_{5}=-c z_{5} ;
\end{aligned}
$$

it is a Hurwitz system and the system is global uniform asymptotic stability. 

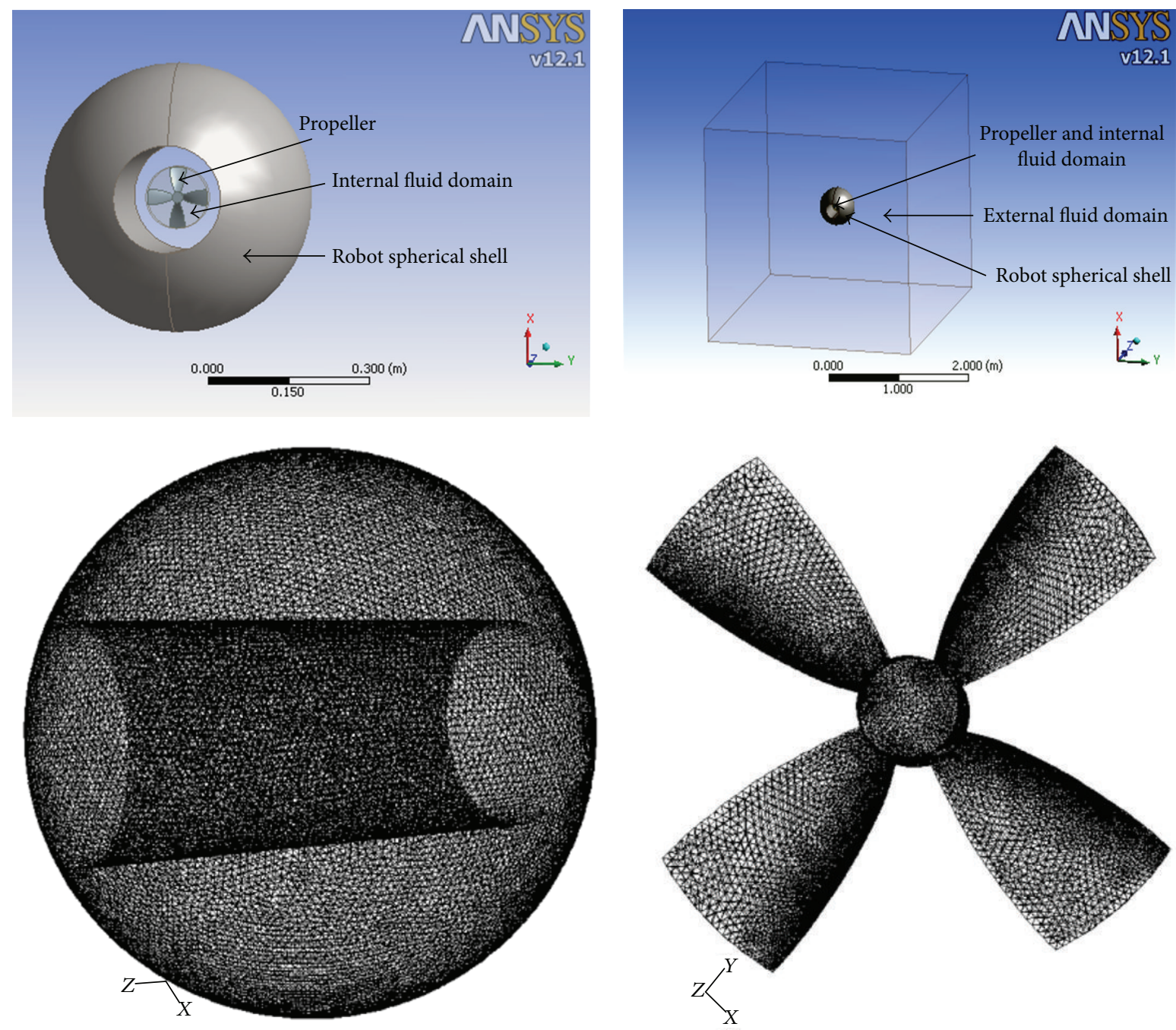

Figure 3: The mesh model of BYSQ-3 for CFD.

\section{System Simulation}

4.1. Control Performance of the Proposed Method. According to the structure of BYSQ-3, the sphere-pipe model is established to simplify the analysis of the inertia mass and hydrodynamic coefficients, and the sphere diameter of BYSQ-3 is $0.3 \mathrm{~m}$. the external fluid domain is a cube with $2 \mathrm{~m}$ side length, and the sphere-pipe model locates in the cube's center. The internal cylindrical fluid domain which contain the propeller is built with a diameter of $0.12 \mathrm{~m}$. the assembly and mesh model of BYSQ-3 are shown in Figure 3. The hydrodynamic coefficients are simulated with the velocity of $1 \mathrm{~m} / \mathrm{s}$. the assembly model is in the center of the external fluid domain with stationary state.

The motion simulations under the above control laws are presented by using the MATLAB programs. The results show that the finite time control laws can steer the BYSQ-3 to the origin fast, accurately, and stably. The control gains are determined by using SIMULINK programs.

$$
\begin{aligned}
& p_{1}=\frac{7}{5}, \\
& \delta_{1}=\frac{2}{5}, \\
& \varepsilon_{1}=\frac{1}{8}, \\
& a_{2}=-5.3, \\
& p_{2}=\frac{37}{25}, \\
& \delta_{2}=\frac{12}{25}, \\
& \varepsilon_{2}=\frac{1}{13} .
\end{aligned}
$$

$$
a_{1}=-17,
$$




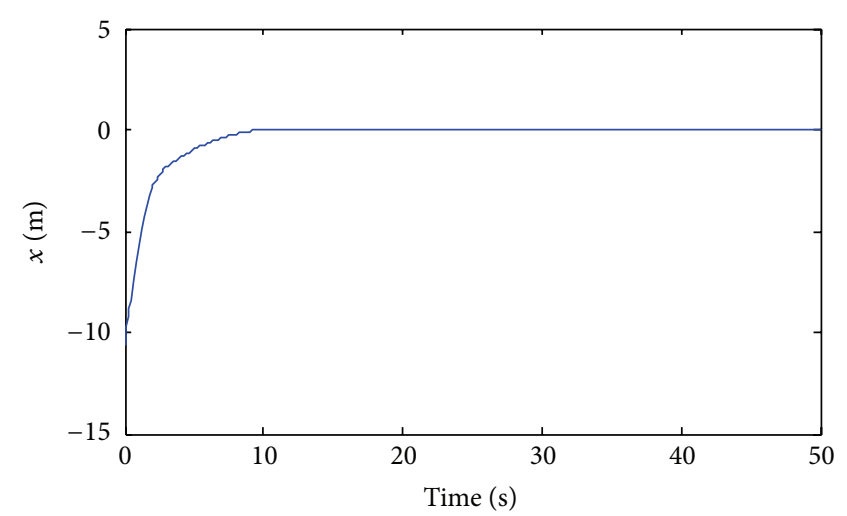

(a)

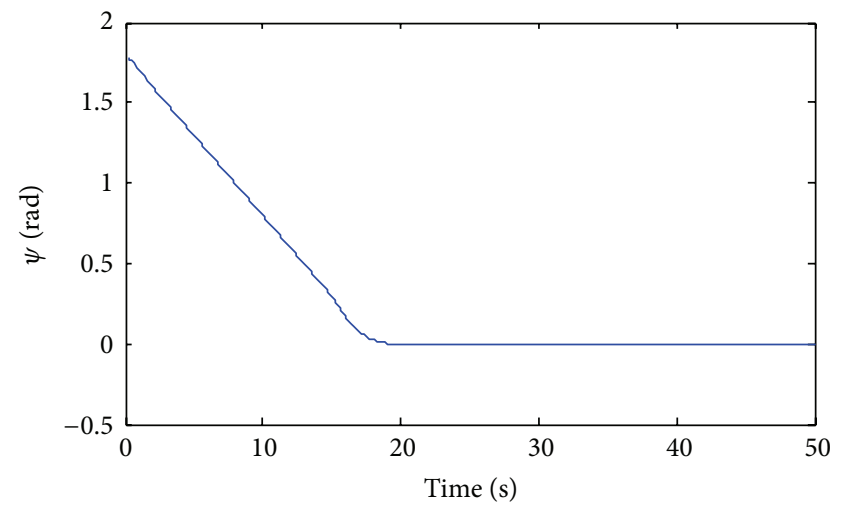

(c)

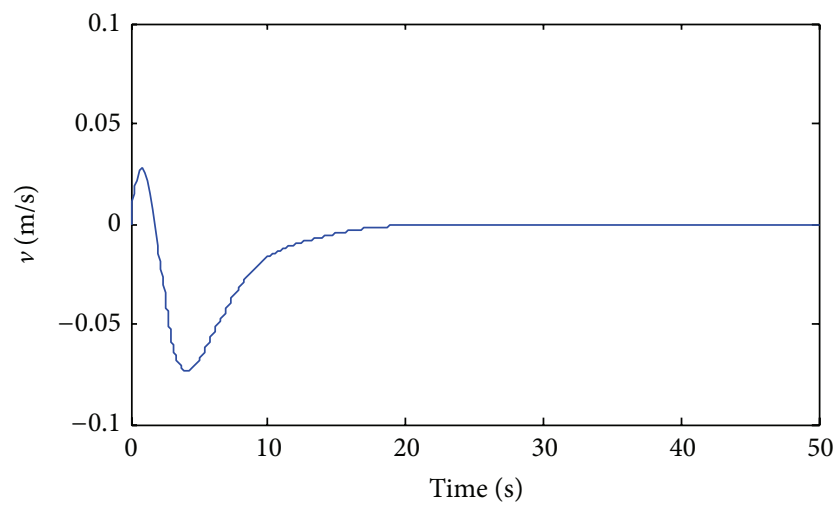

(e)

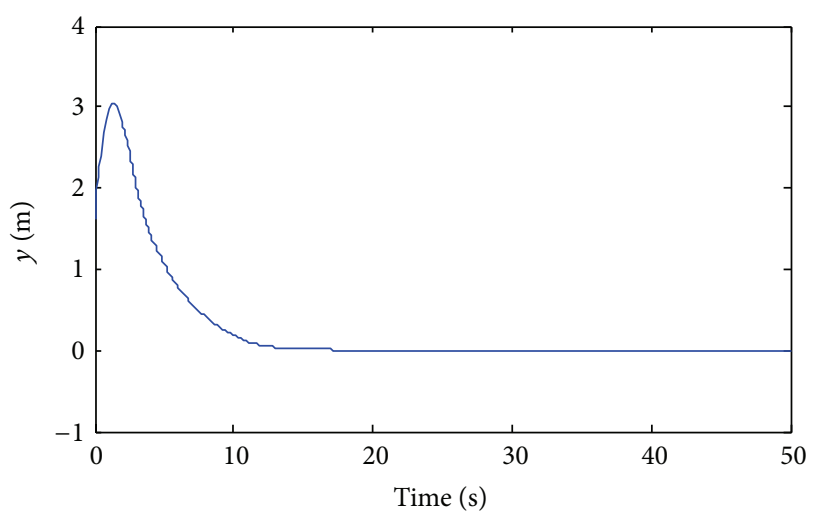

(b)

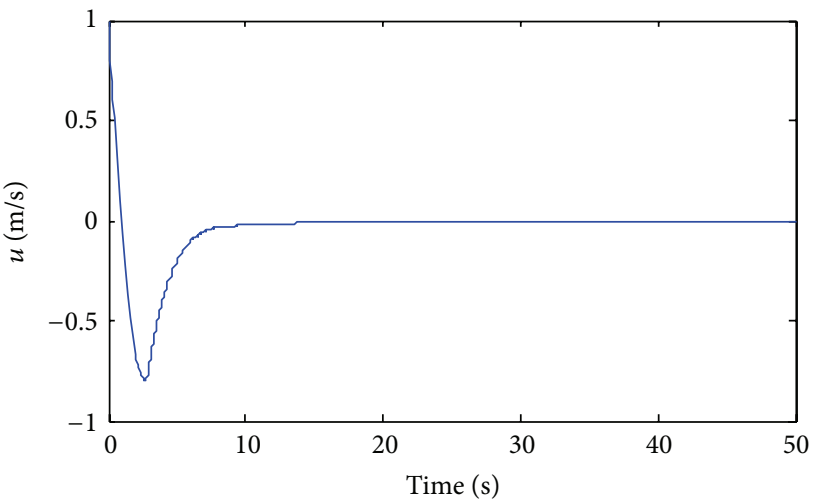

(d)

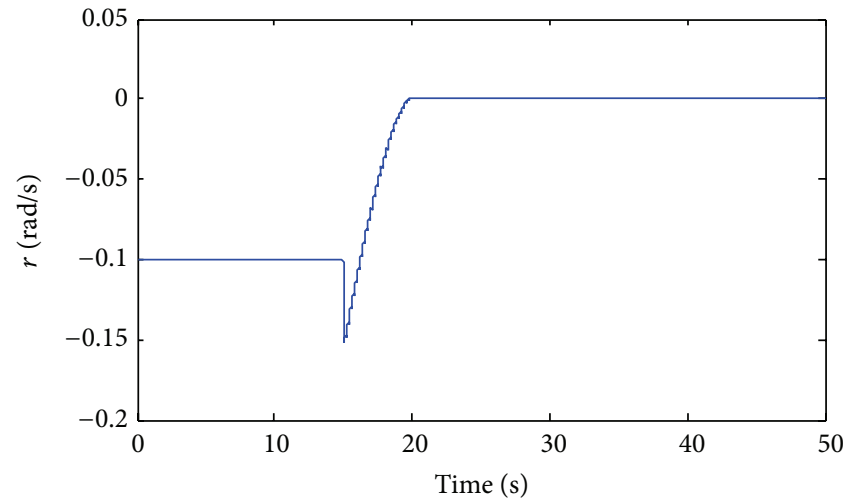

(f)

FIGURE 4: Time history of the state variables.

And the parameters are

$$
\begin{aligned}
& m_{11}=45 \mathrm{~kg}, \\
& m_{22}=46 \mathrm{~kg}, \\
& m_{33}=45.5 \mathrm{~kg}, \\
& d_{11}=0.4 \mathrm{~kg} / \mathrm{s}, \\
& d_{22}=0.2 \mathrm{~kg} / \mathrm{s}, \\
& d_{33}=0.001 \mathrm{~kg} / \mathrm{s} .
\end{aligned}
$$

The initial conditions are

$$
\begin{aligned}
& x(0)=-10.647 \mathrm{~m}, \\
& y(0)=1.65 \mathrm{~m}, \\
& \varphi(0)=1.8 \mathrm{rad}, \\
& u(0)=1 \mathrm{~m} / \mathrm{s}, \\
& v(0)=0 \mathrm{~m} / \mathrm{s}, \\
& r(0)=-0.1 \mathrm{rad} / \mathrm{s} .
\end{aligned}
$$


The time-response of the states is shown in Figure 4. The path traced by BYSQ-3 is shown in Figure 5.

Figures 4 and 5 show the simulations of position and orientation time history of the BYSQ-3; these results show the effectiveness of the proposed finite time control laws. The robot can converge to the origin fast in finite time with desired orientation. The position and orientation errors are less than $0.5 \%$ during steady state. It can be seen that the change slope of the surge velocity is bigger than that of the sway, due to the absence of dependence actuated in the sway direction. Figure 4(c) shows that the change of the angle is uniform at the beginning. This is due to the zero value of $F_{2}$ and the angle velocity being constant; the value of $F_{2}$ begins to change when $t>T_{1}$, so the angle begins to change also; the flywheel steering mechanism is the main innovation of BYSQ-3 which can ensure the efficient and flexible steering.

4.2. Comparison with the Finite Time Controller in [17]. In this subsection, we compared the finite time control law proposed in [17] and the finite time controller proposed in this paper; we adopt the model parameters and initial conditions proposed in [17]:

$$
\begin{aligned}
& x(0)=-3.7142 \mathrm{~m}, \\
& y(0)=-3.8993 \mathrm{~m}, \\
& \varphi(0)=2 \mathrm{rad}, \\
& u(0)=1 \mathrm{~m} / \mathrm{s}, \\
& v(0)=3 \mathrm{~m} / \mathrm{s}, \\
& r(0)=-4 \mathrm{rad} / \mathrm{s} .
\end{aligned}
$$

The gains of the control law proposed in this paper are

$$
\begin{aligned}
& a_{1}=-10.5, \\
& p_{1}=\frac{5}{19}, \\
& \delta_{1}=\frac{2}{7}, \\
& \varepsilon_{1}=\frac{1}{3}, \\
& a_{2}=-6.8, \\
& p_{2}=\frac{7}{19}, \\
& \delta_{2}=\frac{13}{25}, \\
& \varepsilon_{2}=\frac{3}{13} .
\end{aligned}
$$

Figures 6 and 7 show the simulation trajectory, propeller thruster, and steering torque of the controller proposed in
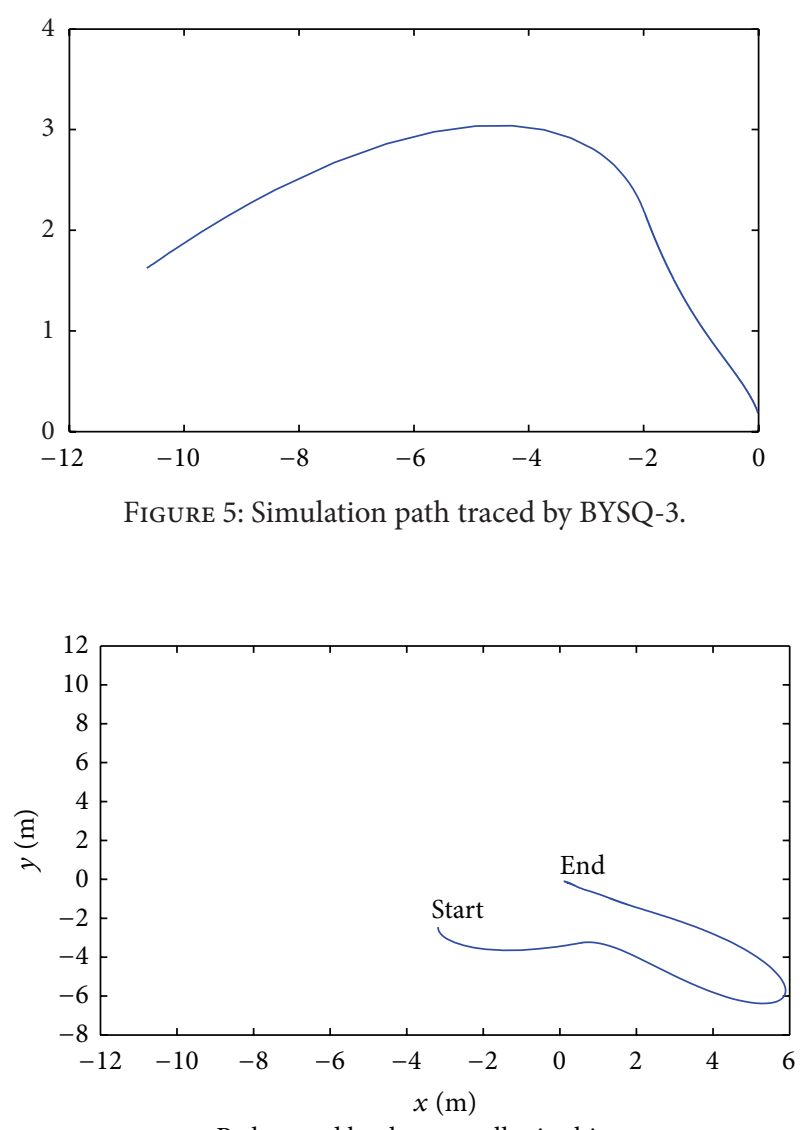

Path traced by the controller in this paper

FIGURE 6: Simulation trajectory comparison of the two controllers.

this paper. Figure 6 shows that the settling-time is more than $100 \mathrm{~s}$ by the controller in [17] (see Figure 4), while it is only $30 \mathrm{~s}$ by controller in this paper; the propeller thruster and steering torque yielded by the controller in [17] (see Figure 4) are about two times larger than those by controller in this paper; this is because, under the effect of the controller in [17] (see Figure 5), the robot yielded a sudden sharp turn while the trajectory curve is smooth by the controllers proposed in this paper. From Figure 6, we can also find that the travelled distance by the controller in [17] (see Figure 5) is more than two times longer than that in this paper. The numerical simulation results show that the controller can be easily generalized to other nonlinear systems and it can save time and energy.

\section{Experiment}

BYSQ-3 is an underwater roving robot prototype with a spherical hull, the diameter is $0.30 \mathrm{~m}$, and the weight of air is about $30.6 \mathrm{~kg}$; see Figure 8 . Compared with BYSQ2, The BYSQ-3 has an improved turning system due to the use of the flywheel mechanism, which is mainly used for aircraft. In order to validate the above control laws proposed in this paper, the experiments have been conducted on the swimming pool in BUPT, China, at the beginning of 


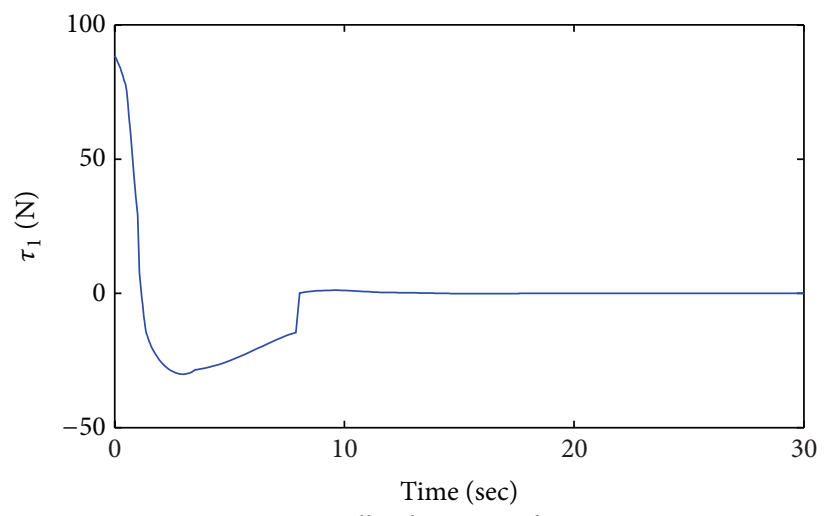

Propeller thruster in this paper

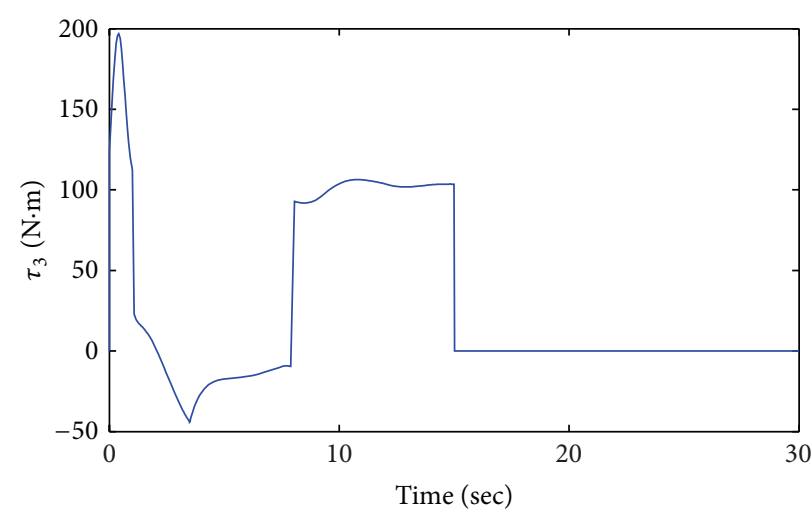

Steering torque in this paper

FIgURE 7: Control torque comparison of the two controllers.
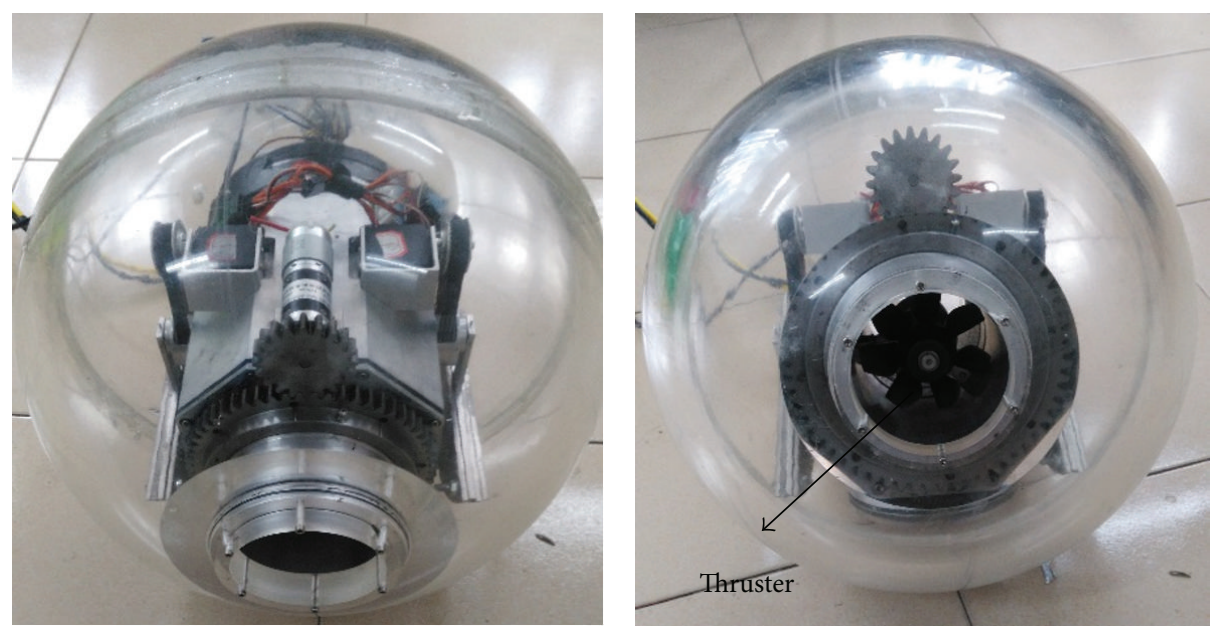

FIgURE 8: The prototype of BYSQ-3.

January 2016. There is no wind in the swimming pool. The experimental setup consists of some sensors (see Figure 9), the BYSQ-3 prototype, and a PC. The robot adopts real-time wireless remote control mode, the internal controller adopts GD32F105 embedded computer running the VxWorks realtime operating system, the sampling frequency for processing navigation and position data is $50 \mathrm{HZ}$, and the communication between the host PC and the internal embedded controller of BYSQ-3 is done via wireless connection. The host computer starts a control period when receiving the robot's motion information and the drive instructions are sent to the internal controller of the robot; the instructions are converted into voltage signal to realize the control task.

Figure 10 is the motion picture of BYSQ-3 in the swimming pool. Figure 11 is the actual trajectory based on the experiment data, and from Figure 10, we can see the distance between the terminal point of BYSQ-3 and the origin is $0.27 \mathrm{~m}$; the result is better than expected.

\section{Conclusion}

In this paper, the finite time control schemes have been proposed to deal with the point stabilization problem of moving the spherical roving robot from one point to another point. The finite time control laws can steer the state variables to origin in finite time, which is better than merely asymptotic convergence. Especially by using the designed control scheme, the coupling degree can be reduced and the convergence rate can be improved. The virtual control inputs do not exist in the design process, which makes it possible to put the controller into practice. Simulation and experimental validation results demonstrate that designed control scheme has good performance; it is time and energy saving control laws with broad application prospects.

\section{Competing Interests}

The authors declare that they have no competing interests. 


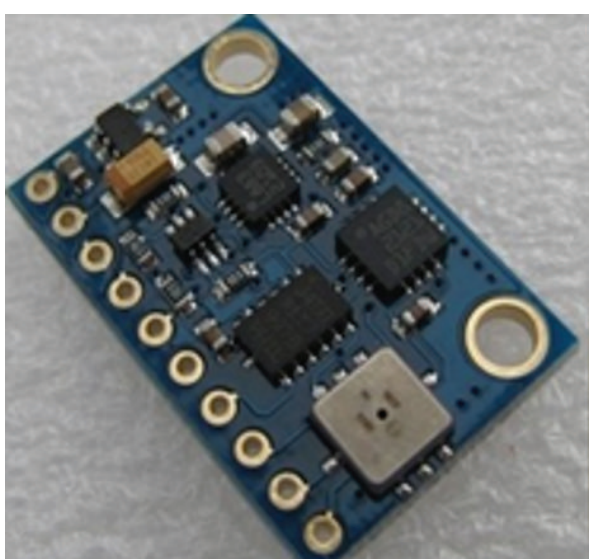

(a) GY-80 nine-axis digital gyroscope

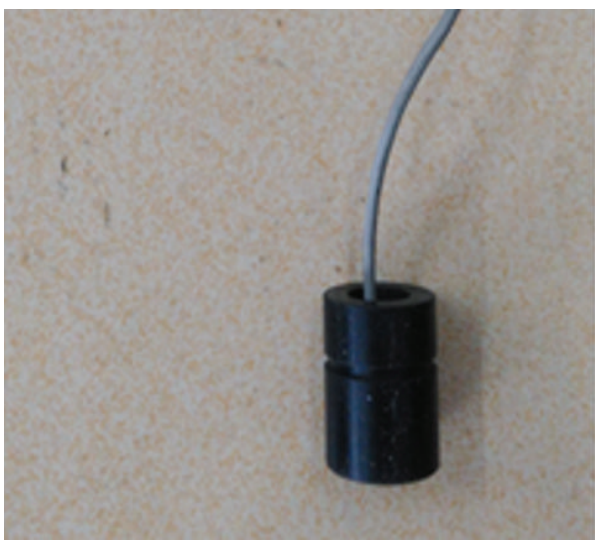

(c) Ultrasonic displacement sensor

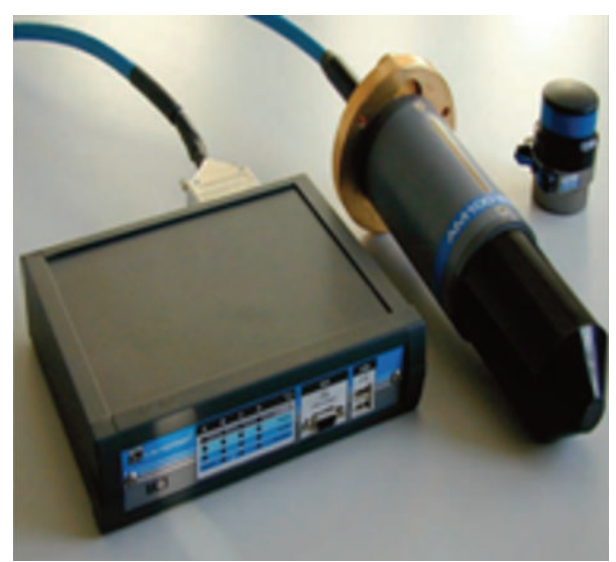

(b) Micronav navigation system

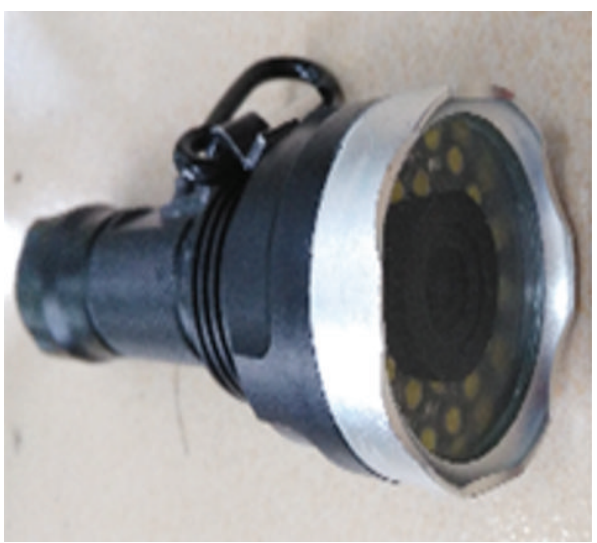

(d) Underwater camera

FIgURE 9: The sensors of BYSQ-3.
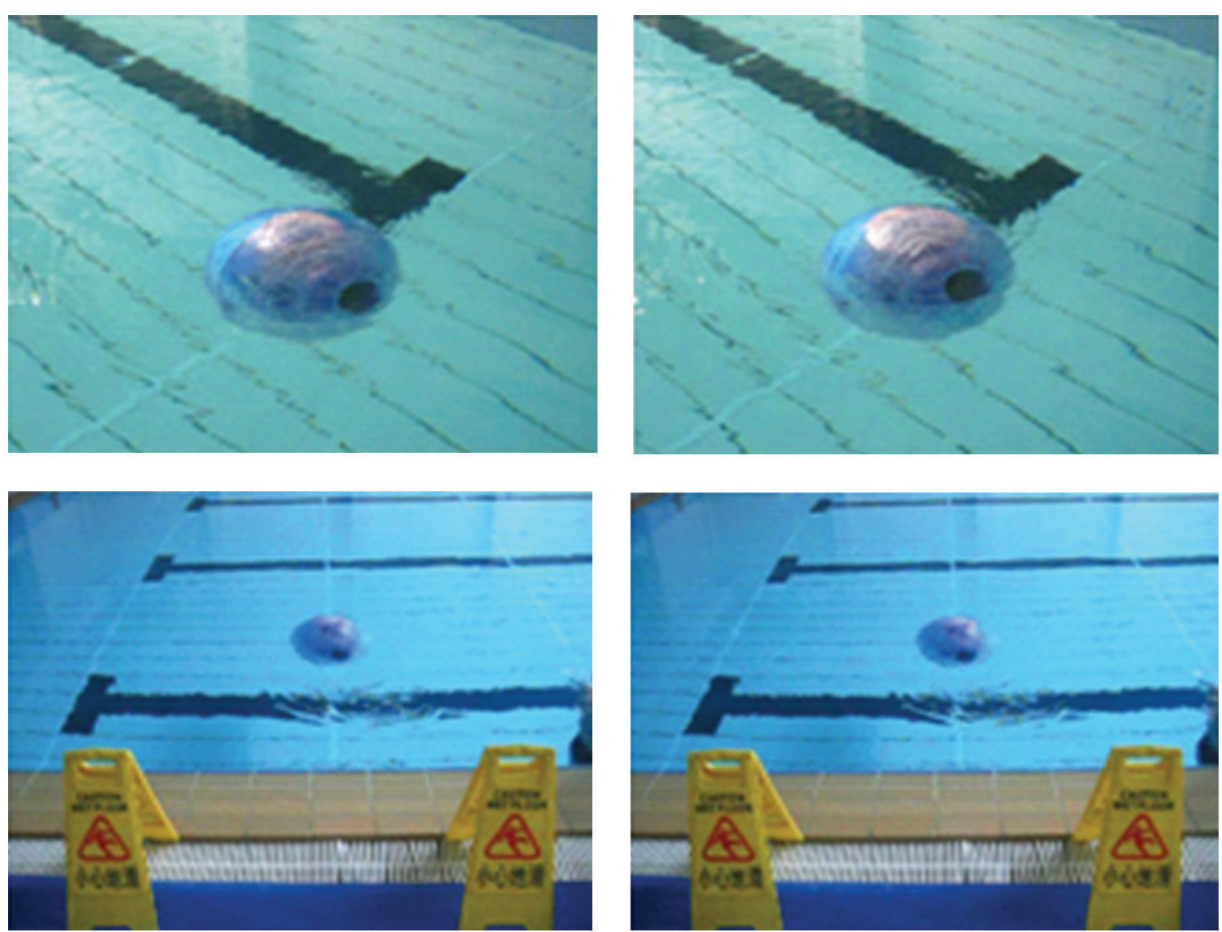

FIGURE 10: Underwater motion of BYSQ-3. 


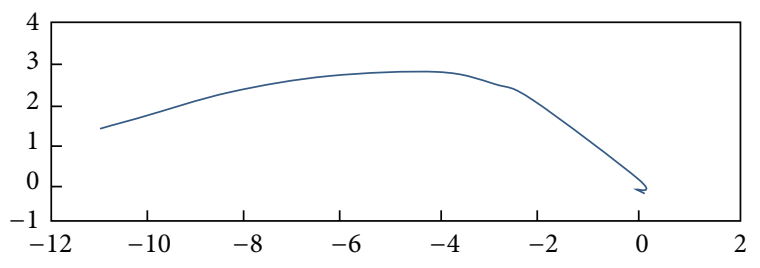

FIGURE 11: Actual motion trajectory of BYSQ-3.

\section{Acknowledgments}

The authors would like to thank the support of China National Natural Science Foundation (51175048) for the research.

\section{References}

[1] M. Bibuli, M. Caccia, L. Lapierre, and G. Bruzzone, "Guidance of unmanned surface vehicles: experiments in vehicle following," IEEE Robotics \& Automation Magazine, vol. 19, no. 3, pp. 92-102, 2012.

[2] F. El-Hawary, The Ocean Engineering Handbook, The Electrical Engineering Handbook, CRC Press, Boca Raton, Fla, USA, 2001.

[3] X. Xiang, L. Lapierre, and B. Jouvencel, "Smooth transition of AUV motion control: from fully-actuated to under-actuated configuration," Robotics and Autonomous Systems, vol. 67, pp. 14-22, 2015.

[4] K. D. Do and J. Pan, Control of Ships and Underwater Vehicles, Advances in Industrial Control, Springer, London, UK, 2009.

[5] R. W. Brockett, "Asymptotic stability and feedback stabilization," Differential Geometric Control Theory, vol. 27, pp. 181-191, 1983.

[6] B.-1. Ma, "Global k-exponential asymptotic stabilization of underactuated surface vessels," Systems \& Control Letters, vol. 58, no. 3, pp. 194-201, 2009.

[7] J. Cheng, J. Q. Yi, and D. B. Zhao, "Stabilization of an underactuated surface vessel via discontinuous control," in Proceedings of the American Control Conference (ACC '07), pp. 206-211, New York, NY, USA, July 2007.

[8] M. Reyhanoglu, "Exponential stabilization of an underactuated autonomous surface vessel," Automatica, vol. 33, no. 12, pp. 2249-2254, 1997.

[9] J. Ghommam, F. Mnif, A. Benali, and N. Derbel, "Asymptotic backstepping stabilization of an underactuated surface vessel," IEEE Transactions on Control Systems Technology, vol. 14, no. 6, pp. 1150-1157, 2006.

[10] H. Przemyslaw, "Decoupled PD set-point controller for underwater vehicles," Ocean Engineering, vol. 36, no. 6, pp. 529-534, 2009.

[11] T.-H. Kim, T. Başar, and I. J. Ha, "Asymptotic stabilization of an underactuated surface vessel via logic-based control," in Proceedings of the American Control Conference, pp. 4678-4683, Anchorage, Alaska, USA, May 2002.

[12] A. P. Aguiar and A. M. Pascoal, "Global stabilization of an underactuated autonomous underwater vehicle via logic-based switching," in Proceedings of the 41st IEEE Conference on Decision and Control, vol. 3, pp. 3267-3272, IEEE, December 2002.
[13] B.-L. Ma and W. Huo, "Smooth time-varying uniform asymptotic stabilization of underactuated surface vessels," in Proceedings of the 48th IEEE Conference on Decision and Control, 2009 held jointly with the 2009 28th Chinese Control Conference (CDC/CCC '09), pp. 3137-3141, IEEE, Shanghai, China, 2009.

[14] W. Dong and Y. Guo, "Global time-varying stabilization of underactuated surface vessel," IEEE Transactions on Automatic Control, vol. 50, no. 6, pp. 859-864, 2005.

[15] J. Ghommam, F. Mnif, and N. Derbel, "Global stabilisation and tracking control of underactuated surface vessels," IET Control Theory and Applications, vol. 4, no. 1, pp. 71-88, 2010.

[16] K. Y. Pettersen, F. Mazenc, and H. Nijmeijer, "Global uniform asymptotic stabilization of an underactuated surface vessel: experimental results," IEEE Transactions on Control Systems Technology, vol. 12, no. 6, pp. 891-903, 2004.

[17] V. Sankaranarayanan, A. D. Mahindrakar, and R. N. Banavar, "A switched controller for an underactuated underwater vehicle," Communications in Nonlinear Science \& Numerical Simulation, vol. 13, no. 10, pp. 2266-2278, 2008.

[18] Z. Yan, H. Yu, W. Zhang, B. Li, and J. Zhou, "Globally finitetime stable tracking control of underactuated UUVs," Ocean Engineering, vol. 107, article 3284, pp. 132-146, 2015.

[19] F. Gao, Y. Wu, and Z. Zhang, "Finite-time stabilization of uncertain nonholonomic systems in feedforward-like form by output feedback," ISA Transactions, vol. 59, pp. 125-132, 2015.

[20] Z.-Y. Sun, L.-R. Xue, and K. Zhang, "A new approach to finitetime adaptive stabilization of high-order uncertain nonlinear system," Automatica, vol. 58, pp. 60-66, 2015.

[21] W. Li, X.-J. Xie, and S. Zhang, "Output-feedback stabilization of stochastic high-order nonlinear systems under weaker conditions," SIAM Journal on Control \& Optimization, vol. 49, no. 3, pp. 1262-1282, 2011.

[22] S. P. Bhat and D. S. Bernstein, "Finite-time stability of continuous autonomous systems," SIAM Journal on Control and Optimization, vol. 38, no. 3, pp. 751-766, 2000.

[23] M. Ghasemi, S. G. Nersesov, and G. Clayton, "Finite-time tracking using sliding mode control," Journal of the Franklin Institute, vol. 351, no. 5, pp. 2966-2990, 2014.

[24] M. Kryachkov, A. Polyakov, and V. Strygin, "Finite-time stabilization of an integrator chain using only signs of the state variables," in Proceedings of the 11th International Workshop on Variable Structure Systems (VSS '10), pp. 510-515, Mexico City, Mexico, June 2010.

[25] Z.-Y. Sun and Y.-G. Liu, "Adaptive control design for a class of uncertain high-order nonlinear systems with time delay," Asian Journal of Control, vol. 17, no. 2, pp. 535-543, 2015.

[26] W. Ai, J. Zhai, and S. Fei, "Output feedback stabilization for a class of stochastic high-order feedforward nonlinear systems with time-varying delay," Asian Journal of Control, vol. 17, no. 5, pp. 1631-1642, 2015.

[27] Z.-Y. Sun, Z.-G. Liu, and X.-H. Zhang, "New results on global stabilization for time-delay nonlinear systems with low-order and high-order growth conditions," International Journal of Robust and Nonlinear Control, vol. 25, no. 6, pp. 878-899, 2015.

[28] F. Gao, Y. Wu, and F. Yuan, "Global output feedback stabilisation of high-order nonlinear systems with multiple time-varying delays," International Journal of Systems Science, vol. 47, no. 10, pp. 2382-2392, 2016.

[29] Z.-Y. Sun, X.-H. Zhang, and X.-J. Xie, "Continuous global stabilisation of high-order time-delay nonlinear systems," International Journal of Control, vol. 86, no. 6, pp. 994-1007, 2013. 
[30] Y. Wu, F. Gao, and Z. Liu, "Finite-time state-feedback stabilisation of non-holonomic systems with low-order non-linearities," IET Control Theory and Applications, vol. 9, no. 10, pp. 15531560, 2015.

[31] C. Qian and W. Lin, "Non-Lipschitz continuous stabilizers for nonlinear systems with uncontrollable unstable linearization," Systems \& Control Letters, vol. 42, no. 3, pp. 185-200, 2001.

[32] C. Qian and W. Lin, "A continuous feedback approach to global strong stabilization of nonlinear systems," IEEE Transactions on Automatic Control, vol. 46, no. 7, pp. 1061-1079, 2001.

[33] Y. Hong, J. Huang, and Y. Xu, "On an output feedback finitetime stabilisation problem," in Proceedings of the 38th IEEE Conference on Decision and Control (CDC'99), vol. 2, pp. 13021307, IEEE, Phoenix, Ariz, USA, December 1999.

[34] S. P. Bhat and D. S. Bernstein, "Geometric homogeneity with applications to finite-time stability," Mathematics of Control, Signals, and Systems, vol. 17, no. 2, pp. 101-127, 2005. 


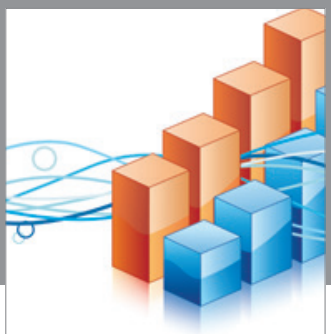

Advances in

Operations Research

vatem alat4

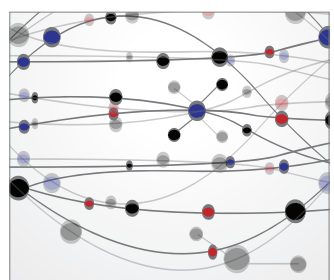

\section{The Scientific} World Journal
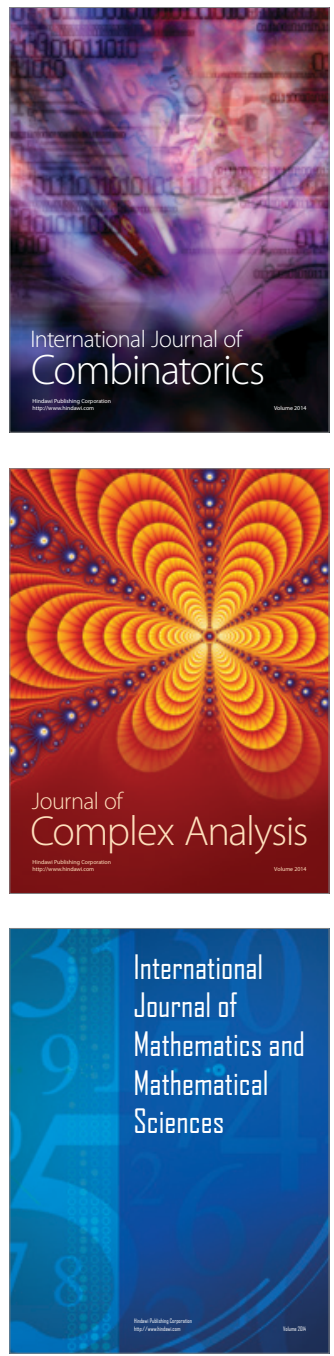
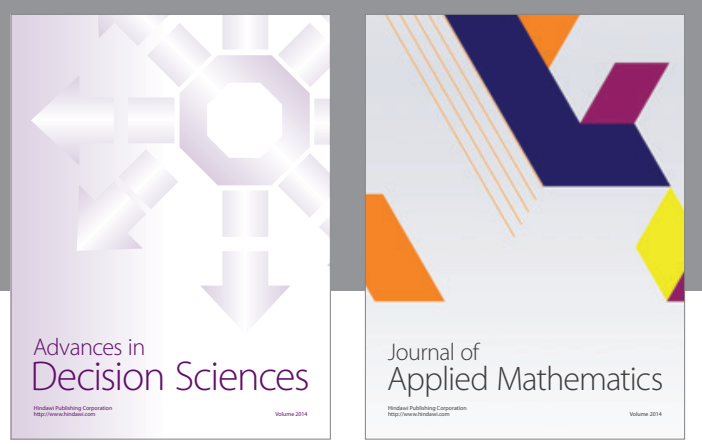

Algebra

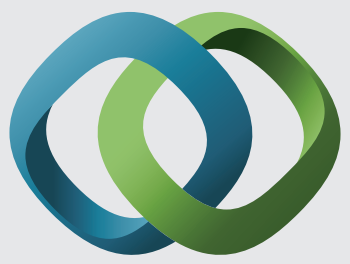

\section{Hindawi}

Submit your manuscripts at

http://www.hindawi.com
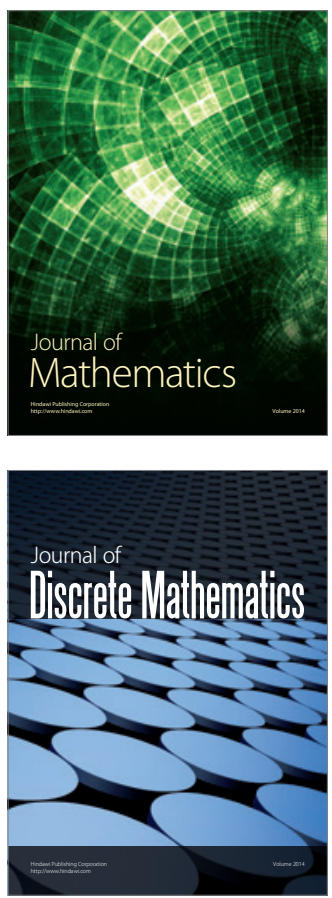

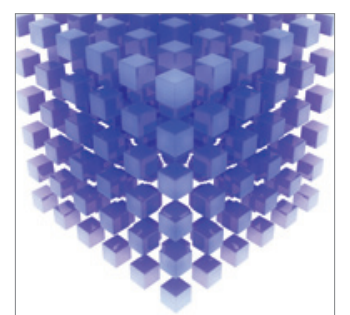

Mathematical Problems in Engineering
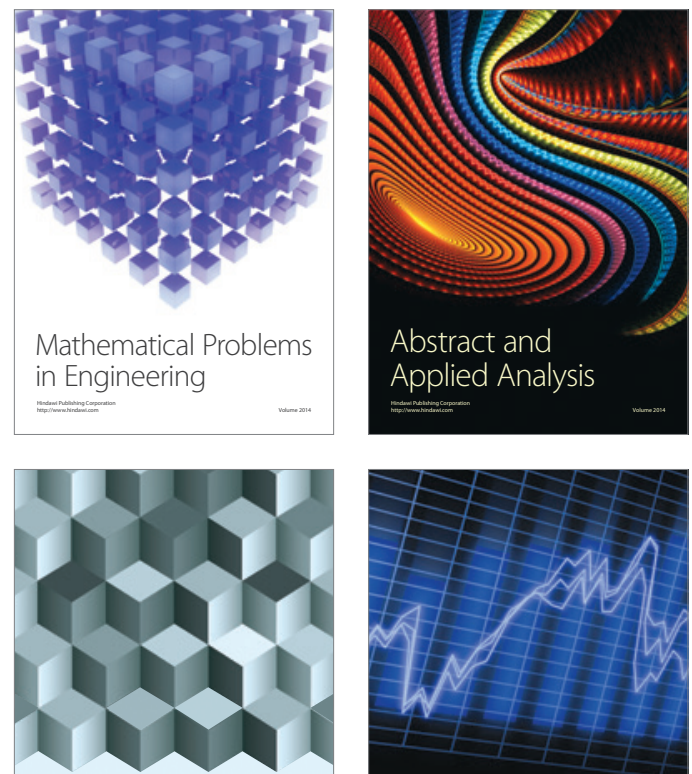

Journal of

Function Spaces

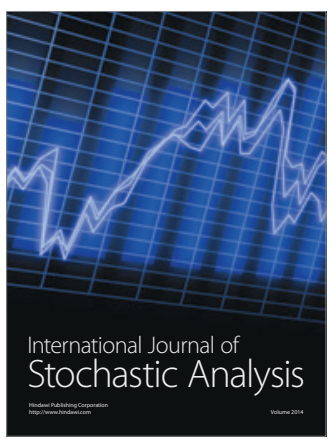

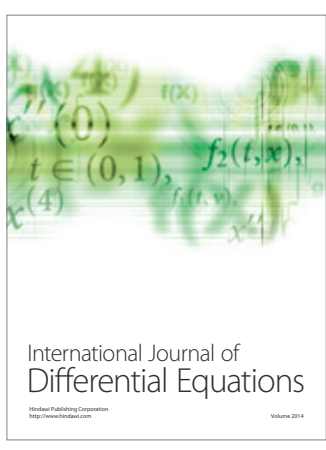
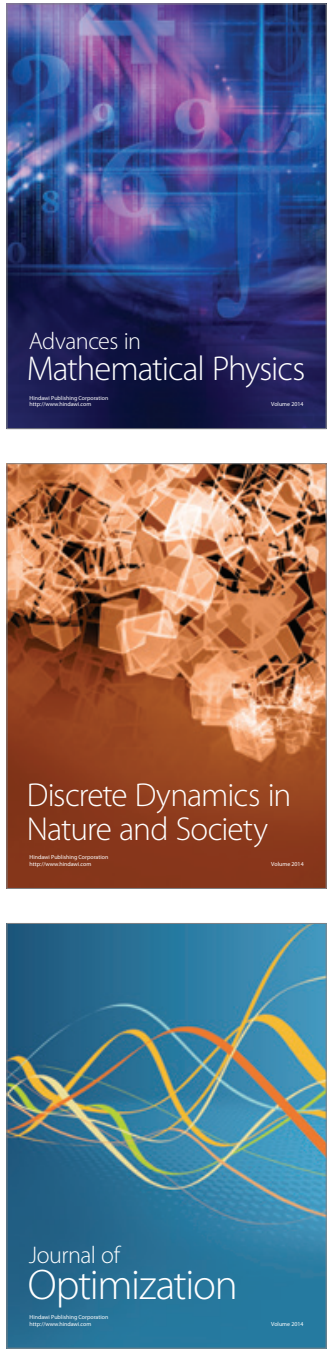